\title{
Comparison of magnetron sputtering and ion beam sputtering on dispersive mirrors
}

\author{
Y. Chen ${ }^{1} \cdot$ D. Hahner ${ }^{2} \cdot$ M. Trubetskov ${ }^{1} \cdot$ S. Schrameyer ${ }^{3} \cdot$ W. Sakiew ${ }^{3} \cdot$ K. Starke ${ }^{3} \cdot$ V. Pervak $^{2}$
}

Received: 31 January 2020 / Accepted: 26 March 2020 / Published online: 15 April 2020

(c) The Author(s) 2020

\begin{abstract}
We have compared two kinds of dispersive mirrors (DMs) produced by magnetron sputtering and ion beam sputtering. One of them is a broadband DM which is known as double-angle DM, providing a group delay dispersion (GDD) of $-40 \mathrm{fs}^{2}$ in the range of 550-1050 $\mathrm{nm}$. The other one is a robust highly dispersive mirror, which provides a GDD of about $-275 \mathrm{fs}^{2}$ at 800 $\mathrm{nm}$ and covers the wavelength range from 690 to $890 \mathrm{~nm}$. For the first time, a comparison between magnetron-sputteringproduced and ion-beam-sputtering-produced dispersive mirrors is performed.
\end{abstract}

\section{Introduction}

Dispersive mirrors (DMs) were first invented by Szipocs et al. [1]. Since 1994, a lot of scientists have been attracted into this field. For the past 2 decades, DMs exploited as intracavity and extracavity dispersion compensation components have been widely used in all kinds of ultrafast laser systems, including Ti:sapphire oscillators [2-5], Yb:YAG disk oscillators [6-8], Erbium-doped fiber chirped pulse amplification systems [9], etc. [10,11]. DMs have covered the wavelength range from the ultraviolet [12], visible [2-5], infrared $[6-11,13,14]$, to mid-infrared region $[15,16]$. Nowadays DMs become the key elements to control dispersion in ultrafast laser systems.

Broadband dispersive mirrors (BBDMs) [15-20] and high-dispersive mirrors (HDMs) [2-9, 13] are the two mostly used DMs. Design and manufacture of both kinds of DMs are the challenge. The first dispersive mirror [1] was fabricated by electron beam evaporation in spite of its relatively low accuracy in layer thickness control. However, as

V. Pervak

vladimir.pervak@physik.uni-muenchen.de

Y. Chen

yu.chen@physik.lmu.de

1 Max-Planck Institute of Quantum Optics, Hans-Kopfermann str. 1, 85748 Garching, Germany

2 Ludwig-Maximilians-Universitaet Muenchen, Am Coulombwall 1, 85748 Garching, Germany

3 Cutting Edge Coatings GmbH, Hannover, Germany more complicated DMs were required to meet the dispersion control in laser systems, electron beam evaporation technique was no longer suitable for producing DMs. Magnetron sputtering (MS) $[6-8,15-20]$ and ion beam sputtering (IBS) [9, 21], which have improved layer thickness accuracy and process stability, became the most widespread coating processes for manufacturing DMs. Both processes deposit layers with excellent uniformity of physical thicknesses and high stability to environmental conditions compared to layers produced by electron beam evaporation. However, there is no study about comparisons of MS-produced and IBSproduced DMs, especially for the two widely used DMs (BBDMs and HDMs).

In this work, we demonstrate the design, production and characterization of (1) double-angle DMs (BBDMs covering one octave) and (2) HDMs. For the first time, the IBS-produced DMs are directly compared with MS-produced DMs. To make the comparison feasible, the same design targets were used in both cases and only fine-tuning was performed to adjust design to refractive indexes of coating plants. In Sect. 2 design, production and characterization of the double angle DMs are presented. We provide design, deposition and characterization of robust HDMs in Sect. 3. A comparison and discussion of the results can be found in Sect. 4. Finally, the conclusion is drawn in Sect. 5. 
Table 1 Cauchy formula coefficients for the layer materials and the Suprasil substrate

\begin{tabular}{llllll}
\hline Technology & Material & $A_{0}$ & $A_{1}$ & $A_{2}$ & $n(800 \mathrm{~nm})$ \\
\hline IBS & $\mathrm{SiO}_{2}$ & 1.486272 & $-3.996783 \mathrm{e}^{-3}$ & $5.8433165 \mathrm{e}^{-4}$ & 1.49 \\
& $\mathrm{Nb}_{2} \mathrm{O}_{5}$ & 2.179779 & 0.032789 & $1.9913311 \mathrm{e}^{-3}$ & 2.24 \\
& $\mathrm{Suprasil}^{*}$ & 1.443268 & 0.004060 & $6.9481764 \mathrm{e}^{-6}$ & 1.45 \\
$\mathrm{MS}$ & $\mathrm{SiO}_{2}$ & 1.465294 & 0.0 & $4.710804 \mathrm{e}^{-4}$ & 1.47 \\
& $\mathrm{Nb}_{2} \mathrm{O}_{5}$ & 2.218485 & 0.021827 & $3.9996753 \mathrm{e}^{-3}$ & 2.26 \\
& Suprasil & 1.443268 & 0.004060 & $6.9481764 \mathrm{e}^{-6}$ & 1.45 \\
\hline
\end{tabular}

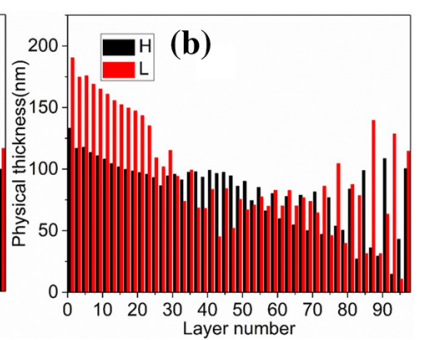

Fig. 1 Layer thicknesses of double-angle DMs: a MS, b IBS. The black and red bars represent the high- and low-index materials, respectively. The layer number starts at the substrate and increases towards the incident medium (air)

\section{Broadband double-angle DMs}

\subsection{Design}

Double-angle DMs were designed by the OptiLayer software [22]. To cover a wide range from 550 to $1050 \mathrm{~nm}, \mathrm{Nb}_{2} \mathrm{O}_{5}$ and $\mathrm{SiO}_{2}$ were chosen as the layer materials. Suprasil was used as the substrate. The optical constants of the substrate and layer materials are specified by the Cauchy formula:

$n(\lambda)=A_{0}+A_{1} / \lambda^{2}+A_{2} / \lambda^{4}$,

where $\lambda$ is expressed in micrometer and the coefficients of $A_{0}, A_{1}$ and $A_{2}$ are presented in Table 1.

$5^{\circ}$ and $19^{\circ}$ were chosen as the two different working angles. The target reflectance was set to 100\% . GDD should compensate $1 \mathrm{~m}$ of Air and $1 \mathrm{~mm}$ of Fused Silica in two reflections with this mirror. The needle optimization and gradual evolution technique [23, 24] were used in the design algorithm [19]. The final optimized thickness structures for MS and IBS are shown in Fig. 1a, b, respectively. The thickness structures are quite similar and there is just a slight difference due to the refractive index differences between the two plants as can be seen in Table 1. The total thickness of both structures is about $8.8 \mu \mathrm{m}$ with 98 layers. Figure 2 depicts the GDD of the double-angle DMs. The GDD oscillations of the individual mirror at two angles of incidence are just in anti-phase, and they cancel out each other. As a result, the effective GDD, which is the arithmetic mean of

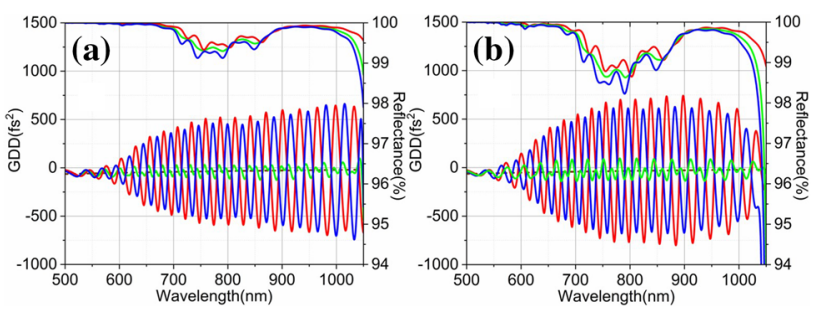

Fig. 2 Theoretical GDD and reflectance of double-angle DMs: a MS, b IBS. The red and blue curves correspond to the incident angle of $5^{\circ}$ and $19^{\circ}$, while the green curves represent the residual GDD and reflectance

the GDD for these two incident angles, has been suppressed significantly.

\subsection{Deposition and characterization}

Both MS and IBS have proven to be the most reliable techniques for producing complicated DMs. The excellent stability and repeatability of sputtering techniques make them the most widely used and successful methods to deposit all kinds of DMs. The double-angle DMs for IBS were manufactured by a NAVIGATOR machine from Cutting Edge Coatings $\mathrm{GmbH}$ (Hannover, Germany). The ion beam is extracted from an Argon gas plasma by a three-grid multi-aperture extraction system. A cryogenic pump evacuates the coating chamber to $1 \times 10^{-7}$ mbar before deposition. The pressure during the coating process is about $5 \times 10^{-4} \mathrm{mbar}$. Due to a stable sputtering rate, the layer thickness was controlled by time. The deposition rates of $\mathrm{Nb}_{2} \mathrm{O}_{5}$ and $\mathrm{SiO}_{2}$ materials were approximately $0.09 \mathrm{~nm} / \mathrm{s}$ and $0.1 \mathrm{~nm} / \mathrm{s}$, respectively. The double-angle DMs for MS were fabricated by a HELIOS machine from Leybold Optics GmbH (Alzenau, Germany). The layer thicknesses were controlled also by time. The system was pumped by turbo-molecular pumps to $1 \times 10^{-6}$ mbar before deposition. The gas pressure was $1 \times 10^{-3} \mathrm{mbar}$ during the sputtering process. The deposition rates were approximately $0.5 \mathrm{~nm} / \mathrm{s}$ for both materials.

The transmittance spectra of the DMs were measured by a spectrophotometer (Perkin Elmer Lambda 950) at normal incidence in the wavelength range of $400-1200 \mathrm{~nm}$. The GDD was measured with a home-built white light interferometer 

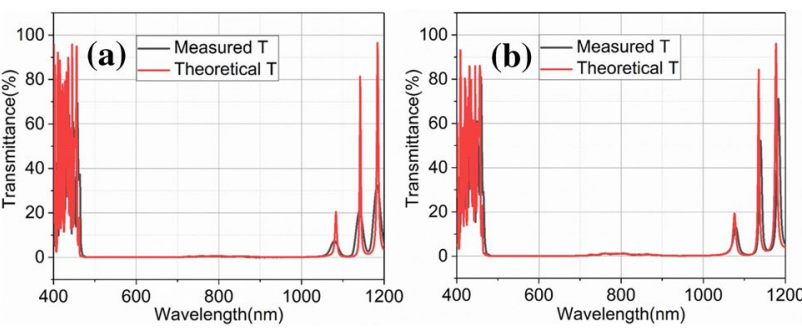

Fig. 3 Measured and theoretical transmittance of double-angle DMs: a MS, b IBS. Black and red curves represent the measured and theoretical transmittance, respectively
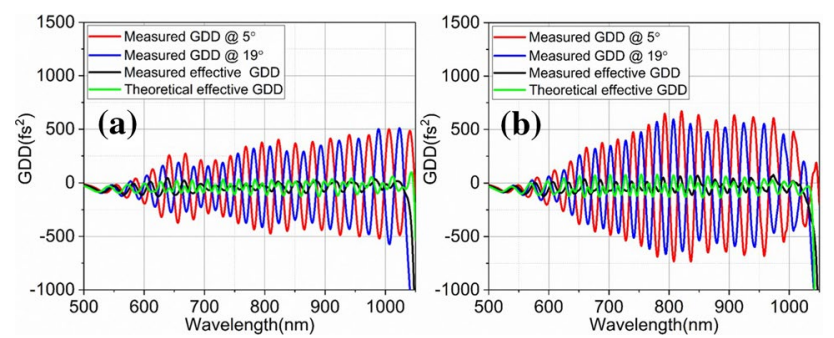

Fig. 4 Measured and theoretical GDD of double-angle DMs: a MS, b IBS. Red and blue curves correspond to the incident angle of $5^{\circ}$ and $19^{\circ}$, black and green curves represent the measured and theoretical effective GDD

[25]. The results of the measured transmittance and GDD can be compared with the theoretical values of the double-angle DMs in Figs. 3 and 4.

Even though a small spectral shift can be observed in the measured transmittance, the measured GDD curves at $5^{\circ}$ and $19^{\circ}$ for both MS-produced and IBS-produced are matching each other, and the GDD oscillations are in anti-phase resulting in the significant decrease of the GDD oscillations for the effective GDD (black curves in Fig. 4). Good agreement between the measured effective GDD and theoretical effective GDD for both IBS and MS processes was obtained.

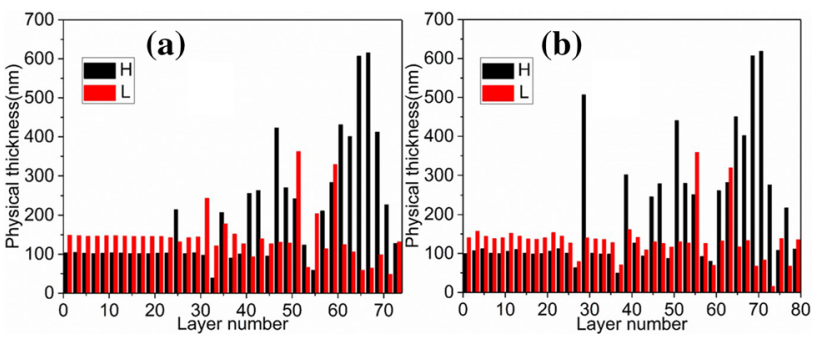

Fig. 5 Layer thicknesses of HDMs: a MS, b IBS. The black and red bars represent the high- and low-index materials, respectively. The layer number starts at the substrate and increases towards the incident medium (air)

\section{Robust HDMs}

\subsection{Design}

For the HDM, the well-known robust synthesis algorithm [26] was used to design the layer structure. It considers the layer thickness discrepancies. $\mathrm{Ta}_{2} \mathrm{O}_{5}$ and $\mathrm{SiO}_{2}$ were chosen as the high and low refractive index materials, while Suprasil was the substrate. The refractive indices of the layer materials and the substrate for both MS and IBS technology were specified by the Cauchy formula. The optical constants are shown in Table 2. By the robust synthesis approach, the number of samples in the cloud was set to 50 [26], which is enough for optimizing the DM according to our experience. The large weight of the absolute errors and small weight of the relative errors were considered. The absolute error was $0.5 \mathrm{~nm}$ and the relative error was zero. The reflectance and GDD values were optimized to $100 \%$ and $-275 \mathrm{fs}^{2}$ at $800 \mathrm{~nm}$ for P-polarization. The theoretical reflectance and GDD for MS and IBS are depicted in Fig. 6. The corresponding thickness structures are shown in Fig. 5. The layer structure for MS consists of 74 layers with a total thickness of $12.6 \mu \mathrm{m}$, whereas the structure for IBS includes 80 alternative high- and low-index layers with a total thickness of $13.2 \mu \mathrm{m}$. The IBS coating is a bit
Table 2 Cauchy formula coefficients for the layer materials and the Suprasil substrate

\begin{tabular}{llllll}
\hline Technology & Material & $A_{0}$ & $A_{1}$ & $A_{2}$ & $n(800 \mathrm{~nm})$ \\
\hline IBS & $\mathrm{SiO}_{2}$ & 1.486272 & $-3.996783 \mathrm{e}^{-3}$ & $5.8433165 \mathrm{e}^{-4}$ & 1.49 \\
& $\mathrm{Ta}_{2} \mathrm{O}_{5}$ & 2.022438 & $1.8653421 \mathrm{e}^{-2}$ & $1.1442954 \mathrm{e}^{-3}$ & 2.05 \\
& $\mathrm{Suprasil}$ & 1.443268 & 0.004060 & $6.9481764 \mathrm{e}^{-6}$ & 1.45 \\
$\mathrm{MS}$ & $\mathrm{SiO}_{2}$ & 1.465294 & 0.0 & $4.710804 \mathrm{e}^{-4}$ & 1.47 \\
& $\mathrm{Ta}_{2} \mathrm{O}_{5}$ & 2.065721 & 0.01683 & 0.001686 & 2.10 \\
& Suprasil & 1.443268 & 0.004060 & $6.9481764 \mathrm{e}^{-6}$ & 1.45 \\
\hline
\end{tabular}



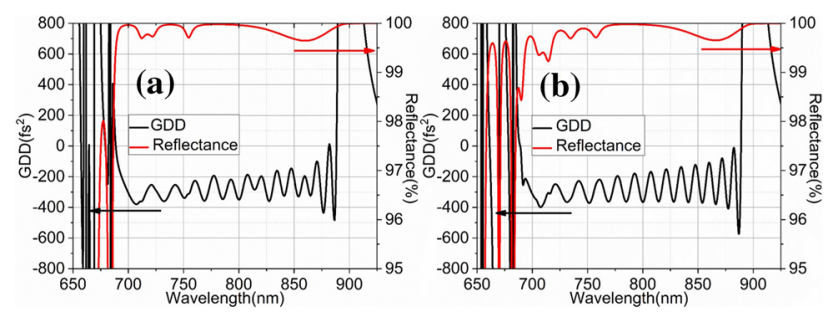

Fig. 6 Theoretical GDD and reflectance of HDMs: a MS, b IBS. Red and black curves correspond to the theoretical reflectance and GDD
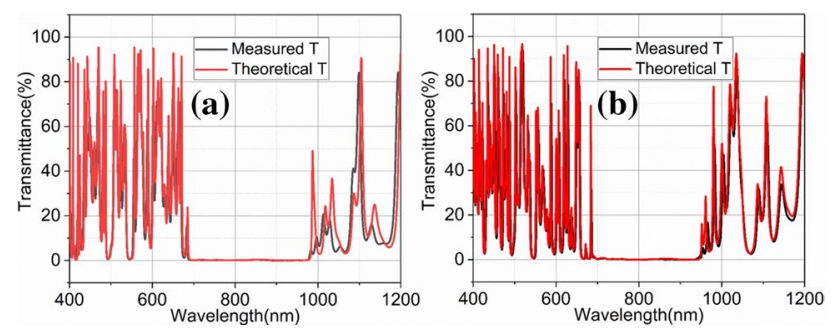

Fig. 7 Measured and theoretical transmittance of HDMs: a MS, b IBS. Black and red curves represent the measured and theoretical transmittance
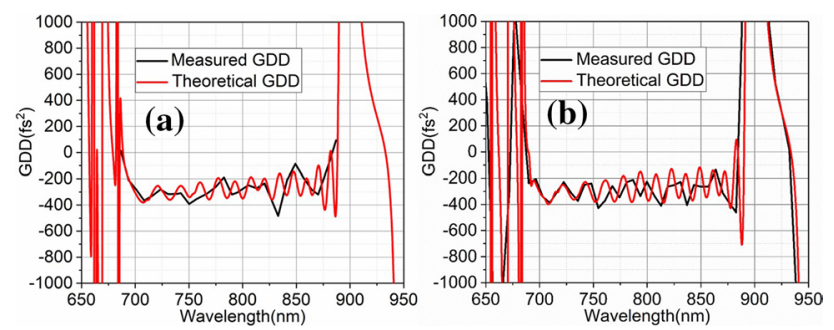

Fig. 8 Measured and theoretical GDD of HMDs: a MS, b IBS. Black and red curves represent the measured and theoretical GDD

thicker due to the slightly smaller refractive indices ratio of the material pair (Fig. 6).

\subsection{Deposition and characterization}

The HDMs were produced by the IBS and MS technology with the same coating process to the double-angle DMs. The deposition rates of $\mathrm{Ta}_{2} \mathrm{O}_{5}$ and $\mathrm{SiO}_{2}$ were about $0.07 \mathrm{~nm} / \mathrm{s}$ and $0.1 \mathrm{~nm} / \mathrm{s}$ for IBS, while the deposition rates were about 0.5 $\mathrm{nm} / \mathrm{s}$ for both materials for MS.

The measured transmittance and GDD compared with theoretical values of the HDMs produced by the two technologies are shown in Figs. 7 and 8.

The transmittance measurements show that better agreement between the measured and the designed transmittance was obtained for the IBS-produced HDMs, especially for the
Table 3 MF values for the broadband double-angle DMs and Robust HDMs

\begin{tabular}{lll}
\hline Technology & $\begin{array}{l}\text { Broadband double-angle } \\
\text { DMs }\end{array}$ & Robust HDMs \\
\hline MS & 16.1 & 12.3 \\
IBS & 19.0 & 9.5 \\
\hline
\end{tabular}
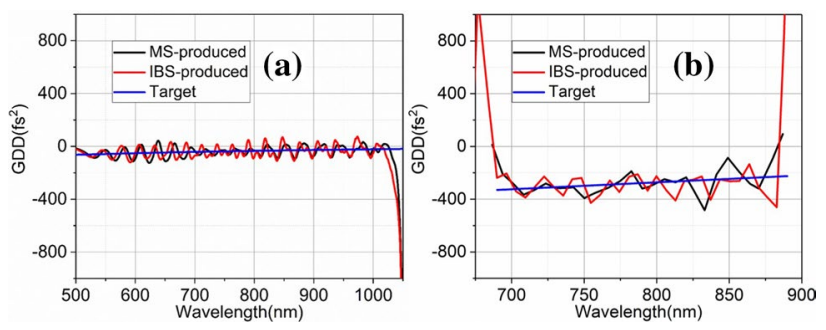

Fig. 9 Measured and theoretical GDD of HMDs: a MS, b IBS. Black and red curves represent the measured and theoretical GDD

longer wavelength region. GDD comparison shows that the measured GDD curves fit very well to the theoretical GDD curves for both coating technologies.

\section{Comparison}

GDD is the most important characteristic of DMs. Thus, the GDD results usually determine whether the coating run is successful or not. To estimate the measured GDD performance, we introduce a merit function to show the discrepancy between the measured GDD and target GDD. The merit function is defined as

$\mathrm{MF}^{2}=\sum_{j=1}^{L}\left(\frac{\operatorname{GDD}\left(m, \lambda_{j}\right)-\operatorname{GDD}\left(T, \lambda_{j}\right)}{\Delta \mathrm{GDD}}\right)^{2}$,

where $\left\{\lambda_{j}\right\}$ are the distributed wavelength points in the spectral range, $\operatorname{GDD}\left(m, \lambda_{j}\right)$ and $\operatorname{GDD}\left(T, \lambda_{j}\right)$ represent the measured GDD and target GDD at the corresponding wavelength, $\Delta$ GDD is the tolerance of GDD. According to formula 2, MF values are calculated, which is shown in Table 3. One can see that for both broadband double-angle DMs and Robust HDMs, the MF values of MS and IBS are very close and relatively small, which means a good agreement between the measured GDD and target GDD.

Measured GDD of double-angle DMs produced by MS and IBS were plotted in Fig. 9a. We can see that both GDD curves have relatively small GDD oscillations and almost the same amount of GDD oscillations. Furthermore, both measured GDD curves meet the target. A comparison of IBS-produced and MS-produced robust HDMs was investigated as 
well, presented in Fig. 9b, which shows that both curves are close to the target and fulfill the GDD requirements. From the GDD comparison, both DMs manufactured by the two technologies have similar GDD characteristics, including the amount of GDD and the number of GDD oscillations. This demonstrates that IBS and MS have a comparable accuracy of the deposited layer thicknesses, by which the produced DMs are composed of.

\section{Conclusion and outlook}

Two different types of DMs have been designed, fabricated and characterized. The comparison of the measured transmittance and GDD with the theoretical values indicates that both DMs produced by MS and IBS are successful. Furthermore, a comparison of the characteristics of the MS-produced and IBS-produced DMs was drawn for the first time. The GDD comparison proves that the IBS technology and the MS technology can produce DMs with same precision.

With the demand of high-power laser systems increasing, large-scale and low loss dielectric coatings, including highly reflective mirrors as well as dispersive mirrors, draw more and more scientist's attention. The IBS is able to deposit mirrors up to a diameter of $600 \mathrm{~mm}$ with a uniformity better than $1 \%$. Ultra-low loss mirrors have been successfully produced with a total loss value of $1 \mathrm{ppm}$. The large coating area and the achievable loss values are the two main advantages of the IBS technology compared to the MS technology. These two advantages as well as the high deposition accuracy make IBS more suitable and promising for producing large size, low loss and complicated dispersive mirrors in the future. The MS technology is significantly faster (roughly by factor 5) and can coat more substrates with smaller size (10 pcs of up to $200 \mathrm{~mm}$ in diameter) in comparison to only one 200-mm substrate in the case of IBS technology.

Acknowledgements Open access funding provided by Projekt DEAL. This work is supported by DFG Cluster of Excellence, Munich Centre for Advanced Photonics. The authors thank Ferenc Krausz for valuable discussion and permanent support of this work.

Open Access This article is licensed under a Creative Commons Attribution 4.0 International License, which permits use, sharing, adaptation, distribution and reproduction in any medium or format, as long as you give appropriate credit to the original author(s) and the source, provide a link to the Creative Commons licence, and indicate if changes were made. The images or other third party material in this article are included in the article's Creative Commons licence, unless indicated otherwise in a credit line to the material. If material is not included in the article's Creative Commons licence and your intended use is not permitted by statutory regulation or exceeds the permitted use, you will need to obtain permission directly from the copyright holder. To view a copy of this licence, visit http://creativecommons.org/licenses/by/4.0/.

\section{References}

1. R. Szipöcs, K. Ferencz, C. Spielmann, F. Krausz, Chirped multilayer coatings for broadband dispersion control in femtosecond lasers. Opt. Lett. 19(3), 201-203 (1994)

2. S.R.A. Dods, Z. Zhang, M. Ogura, Highly dispersive mirror in ta 2 o $5 /$ sio 2 for femtosecond lasers designed by inverse spectral theory. Appl. Opt. 38(21), 4711-4719 (1999)

3. V. Pervak, C. Teisset, A. Sugita, S. Naumov, F. Krausz, A. Apolonski, High-dispersive mirrors for femtosecond lasers. Opt. Express 16(14), 10220-10233 (2008)

4. V. Pervak, I. Ahmad, S.A. Trushin, A.Z. Major, S.K. Apolonski, F. Krausz, Chirped-pulse amplification of laser pulses with dispersive mirrors. Opt. Express 17(21), 19204-19212 (2009)

5. P. Dombi, P. Rácz, M. Lenner, V. Pervak, F. Krausz, Dispersion management in femtosecond laser oscillators with highly dispersive mirrors. Opt. Express 17(22), 20598-20604 (2009)

6. V. Pervak, O. Pronin, J. Olga Razskazovskaya, I.B.Angelov Brons, M.K. Trubetskov, A.V. Tikhonravov, Ferenc Krausz, High-dispersive mirrors for high power applications. Opt. Express 20(4), 4503-4508 (2012)

7. E. Fedulova, K. Fritsch, J. Brons, O. Pronin, T. Amotchkina, M. Trubetskov, F. Krausz, V. Pervak, Highly-dispersive mirrors reach new levels of dispersion. Opt. Express 23(11), 1378813793 (2015)

8. O. Pronin, C.J. Brons, V.P. Grasse, G. Boehm, M.-C. Amann, A. Apolonski, V.L. Kalashnikov, F. Krausz, High-power kerr-lens mode-locked yb: Yag thin-disk oscillator in the positive dispersion regime. Opt. Lett. 37(17), 3543-3545 (2012)

9. Y. Chen, Y. Wang, L. Wang, M. Zhu, H. Qi, J. Shao, X. Huang, S. Yang, C. Li, K. Zhou et al., High dispersive mirrors for erbium-doped fiber chirped pulse amplification system. Opt. Express 24(17), 19835-19840 (2016)

10. H.-W. Chen, T. Sosnowski, C.-H. Liu, L.-J. Chen, J.R. Birge, A. Galvanauskas, F.X. Kärtner, G. Chang, Chirally-coupled-core yb-fiber laser delivering 80 -fs pulses with diffraction-limited beam quality warranted by a high-dispersion mirror based compressor. Opt. Express 18(24), 24699-24705 (2010)

11. P. Wnuk, P. Wasylczyk, M. Dems, K. Hejduk, K. Regiński, A. Wójcik-Jedlińska, A. Jasik et al., Continuously tunable yb:Kyw femtosecond oscillator based on a tunable highly dispersive semiconductor mirror. Opt. Express 22(15), 18284-18289 (2014)

12. M.T.O. Razskazovskaya, T.T. Hassan, E.G. Luu, V. Pervak, Efficient broadband highly dispersive hfo $2 /$ sio 2 multilayer mirror for pulse compression in near ultraviolet. Opt. Express 24(12), 13628-13633 (2016)

13. T. Amotchkina, M. Trubetskov, F. Habel, Y. Pervak, J. Zhang, K. Mak, O. Pronin, F. Krausz, V. Pervak, Synthesis, fabrication and characterization of a highly-dispersive mirrors for the $2 \mu \mathrm{m}$ spectral range. Opt. Express 25(9), 10234-10240 (2017)

14. S. Vasilyev, I. Moskalev, M. Mirov, S. Mirov, V. Gapontsev, Multi-watt mid-ir femtosecond polycrystalline cr 2+: Zns and cr2+: Znse laser amplifiers with the spectrum spanning 2.0-2.6 $\mu \mathrm{m}$. Opt. Express 24(2), 1616-1623 (2016)

15. F. Habel, V. Pervak, Dispersive mirror for the mid-infrared spectral range of 9-11.5 $\mu \mathrm{m}$. Appl. Opt. 56(4), C71-C74 (2017)

16. V. Pervak, T. Amotchkina, Q. Wang, O. Pronin, K.F. Mak, M. Trubetskov, 2/3 octave si/sio 2 infrared dispersive mirrors open new horizons in ultrafast multilayer optics. Opt. Express 27(1), 55-62 (2019)

17. V. Pervak, A.V. Tikhonravov, M.K. Trubetskov, S. Naumov, F. Krausz, A. Apolonski, 1.5-octave chirped mirror for pulse compression down to sub-3 fs. Appl. Phys. B 87(1), 5-12 (2007) 
18. V. Pervak, I. Ahmad, J. Fulop, M.K. Trubetskov, A.V. Tikhonravov, Comparison of dispersive mirrors based on the timedomain and conventional approaches, for sub-5-fs pulses. Opt. Express 17(4), 2207-2217 (2009)

19. V. Pervak, M.K.I. Ahmad, A.V.T. Trubetskov, F. Krausz, Double-angle multilayer mirrors with smooth dispersion characteristics. Opt. Express 17(10), 7943-7951 (2009)

20. F. Habel, V. Shirvanyan, M. Trubetskov, C. Burger, A. Sommer, M.F. Kling, M. Schultze, V. Pervak, Octave spanning wedge dispersive mirrors with low dispersion oscillations. Opt. Express 24(9), 9218-9223 (2016)

21. F.X. Kärtner, N. Matuschek, T. Schibli, U. Keller, H.A. Haus, C. Heine, R. Morf, V. Scheuer, M. Tilsch, T. Tschudi, Design and fabrication of double-chirped mirrors. Opt. Lett. 22(11), 831-833 (1997)

22. A. V. Tikhonravov, M. K. Trubetskov. Optilayer. https://www. optilayer.com/ (1992)

23. A.V. Tikhonravov, M.K. Trubetskov, G.W. DeBell, Application of the needle optimization technique to the design of optical coatings. Appl. Opt. 35(28), 5493-5508 (1996)
24. A.V. Tikhonravov, M.K. Trubetskov, Modern design tools and a new paradigm in optical coating design. Appl. Opt. 51(30), 7319-7332 (2012)

25. T.V. Amotchkina, A.V. Tikhonravov, M.K. Trubetskov, D. Grupe, A. Apolonski, V. Pervak, Measurement of group delay of dispersive mirrors with white-light interferometer. Appl. Opt. 48(5), 949-956 (2009)

26. V. Pervak, M.K. Trubetskov, A.V. Tikhonravov, Robust synthesis of dispersive mirrors. Opt. Express 19(3), 2371-2380 (2011)

Publisher's Note Springer Nature remains neutral with regard to jurisdictional claims in published maps and institutional affiliations. 